\title{
Emission from Charge Recombination during the Pulse Radiolysis of 9-Cyano-10-phenylethynylanthracenes with Donor and Acceptor Substituents
}

\author{
Shingo Samori, ${ }^{\dagger}$ Sachiko Tojo, ${ }^{\dagger}$ Mamoru Fujitsuka, ${ }^{\dagger}$ Hua-Jyu Liang, ${ }^{\ddagger}$ Tong-Ing Ho,,$^{\ddagger}$ \\ Jye-Shane Yang, ${ }^{\ddagger}$ and Tetsuro Majima ${ }^{\dagger, *}$
}

The Institute of Scientific and Industrial Research (SANKEN), Osaka University, Mihogaoka 8-1, Ibaraki, Osaka 567-0047, Japan, and Department of Chemistry, National Taiwan University, Taipei, Taiwan 10617

majima@sanken.osaka-u.ac.jp

Received May 4, 2006
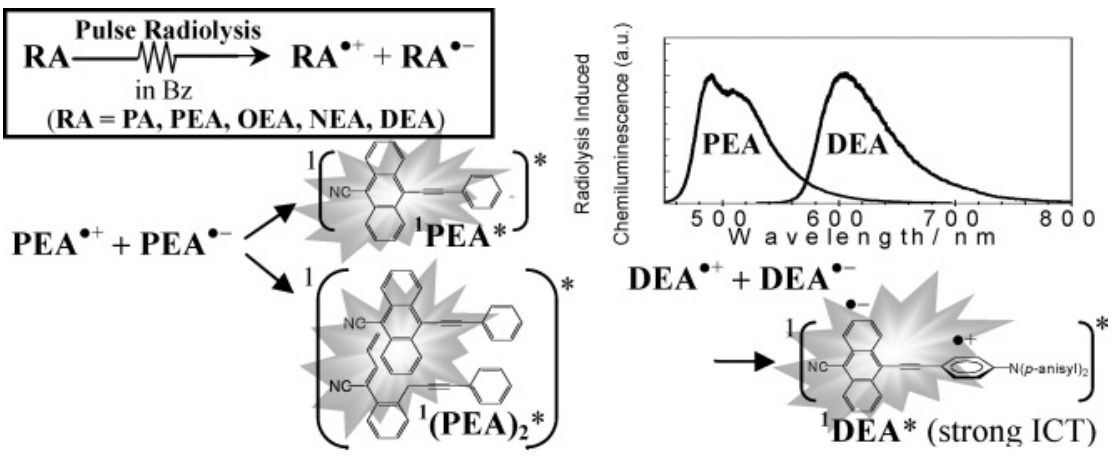

Emission from 9-cyano-10-phenylanthracene and 9-cyano-10-phenylethynylanthracenes having donor and acceptor substituents (RA = PA, PEA, OEA, NEA, and DEA) was studied with the time-resolved fluorescence measurement during the pulse radiolysis of RAs in benzene (Bz). PA and DEA showed only monomer emission, while other RAs (PEA, OEA, and NEA) showed both monomer and excimer emissions with much lower intensities. On the basis of the steady-state and transient absorption and emission measurements, the formation of $\mathbf{R A}$ in the singlet excited state $\left({ }^{1} \mathbf{R} \mathbf{A}^{*}\right)$ can be attributed to the charge recombination between $\mathbf{R A}$ radical cation and anion $\left(\mathbf{R A}^{\bullet+}\right.$ and $\mathbf{R} \mathbf{A}^{\bullet-}$, respectively) which are initially generated from the radiolytic reaction in Bz. It is expected that for PA with a twisted geometry, the charge recombination between $\mathbf{P A}^{\bullet+}$ and $\mathbf{P A}{ }^{\bullet-}$ occurs to give ${ }^{1} \mathbf{P A}^{*}$ during the pulse radiolysis in $\mathrm{Bz}$. For PEA and OEA, $\pi$-stacking interaction is possible for the formation of an encounter complex during the charge recombination between $\mathbf{R A}^{\bullet+}$ and $\mathbf{R A}^{\bullet-}$. For NEA, it is expected that NEA ${ }^{-+}$and NEA$^{--}$ collide neck-to-neck to generate the excimer due to the twisted geometry. For DEA, a considerably twisted structure is assumed to give ${ }^{1} \mathbf{D E A}^{*}$ with strong ICT character but not ${ }^{1}(\mathbf{D E A})_{2}{ }^{*}$ because of the bulky donor substituent.

\section{Introduction}

Charge recombination of radical cation $\left(\mathbf{M}^{\bullet+}\right)$ and anion $\left(\mathbf{M}^{-}\right)$, which are generated by electrolysis of organic molecule (M) in solution, leads to the formation of electronically excited states of $\mathbf{M}$ such as ${ }^{1} \mathbf{M}^{*}$ and/or the singlet excimer of ${ }^{1} \mathbf{M}^{*}\left({ }^{1} \mathbf{M}_{2}{ }^{*}\right)$ on the electrode surfaces. Since ${ }^{1} \mathbf{M}^{*}$ and ${ }^{1} \mathbf{M}_{2}{ }^{*}$ emit the

* To whom correspondence should be addressed. Telephone: Japan +6-68798495. Fax: Japan +6-6879-8499.

Osaka University.

* National Taiwan University. fluorescence, it is called electrogenerated chemiluminescence (ECL). ${ }^{1-5}$ The formation of excited states in the solid state by the application of an electric field (as in OLED) has been shown to follow a mechanism similar to that in solution (ECL). ${ }^{4}$ Recently, organic $\pi$-conjugated donor-acceptor (D-A) type $\mathbf{M}$ has attracted considerable attention as electroluminescent

(1) (a) Faulkner, L. R.; Bard, A. J. Electroanalytical Chemistry; Marcel Dekker: New York, 1977; Vol. 10, pp 1-95. (b) Bard, A. J.; Faulkner, L. R. Electrochemical Methods Fundamentals and Applications, $2^{\text {nd }}$ ed.; John Wiley and Sons: New York, 2001, pp 736-745. (c) Richter, M. M. Chem. Rev. 2004, 104, 3003. 
<smiles>N#Cc1c2ccccc2c(-c2ccccc2)c2ccccc12</smiles>

PA

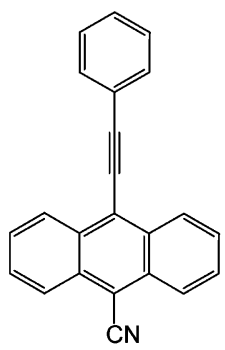

PEA

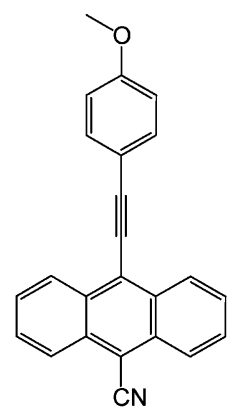

OEA

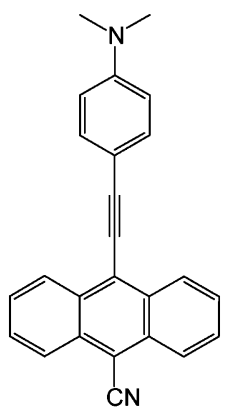

NEA

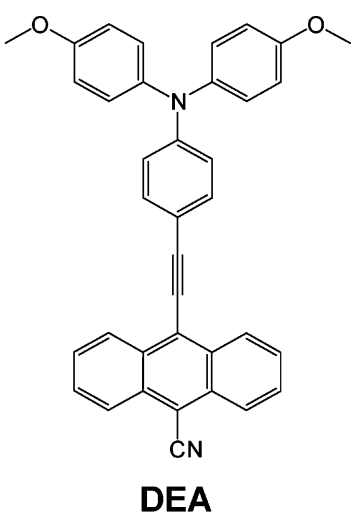

materials for OLEDs. ${ }^{6} \mathrm{ECL}$ from the $\mathrm{D}-\mathrm{A}$ type $\mathbf{M}$ can originate from the locally excited states of D or A moiety, the intramolecular charge transfer (ICT) states, ${ }^{2-3,5 a-c}$ or even from intermolecular excimers or exciplexes. ${ }^{2-3,5 \mathrm{~d}, \mathrm{e}}$ Thus, a judicious choice of the D/A unit could allow the control of the HOMO/ LUMO levels and the emission color of the D-A type $\mathbf{M}$. However, most luminescent materials are highly emissive in their dilute solutions but become weakly luminescent when in concentrated solutions or fabricated into thin films. Under these conditions, the molecules aggregate to form less emissive species such as excimers, leading to a reduction in the luminescence efficiency. When the Stokes shift is small, the luminescence efficiency is also reduced due to self-absorption as a result of high concentration of $\mathbf{M}$ needed to obtain the measurable emission. For some D-A type Ms, the ICT ECL was observed at longer wavelengths than that from the locally excited state, reducing the self-absorption. ${ }^{2,3}$

In our previous papers, we proposed the emission mechanisms of D-A type $\mathbf{M}$ with an ethynyl linkage (RE) such as donorsubstituted phenylquinolinylethynes, ${ }^{7 a}$ phenyl(9-acridinyl)ethynes ${ }^{7 \mathrm{~b}}$ phenyl $\left(9\right.$-cyanoanthracenyl)ethynes, ${ }^{7 \mathrm{~b}}$ and arylethynylpyrenes $^{7 \mathrm{c}}$ during pulse radiolysis in benzene $(\mathrm{Bz})$. Some of these compounds are known as ECL-active $\mathbf{M} .^{2}$ On the basis of the

(2) (a) Elangovan, A.; Chen, T.-Y.; Chen, C.-Y.; Ho, T.-I. Chem. Commun. 2003, 2146. (b) Elangovan, A.; Yang, S.-W.; Lin, J.-H.; Kao, K.-M.; Ho, T.-I. Org. Biomol. Chem. 2004, 2, 1597. (c) Elangovan, A.; Chiu, H.-H.; Yang, S.-W.; Ho, T.-I. Org. Biomol. Chem. 2004, 2, 3113. (d) Elangovan, A.; Kao, K.-M.; Yang, S.-W.; Chen, Y.-L.; Ho, T.-I.; Su, Y. O. J. Org. Chem. 2005, 70, 4460. (e) Yang, S.-W.; Elangovan, A.; Hwang, K.-C.; Ho, T.-I. J. Phys. Chem. B 2005, 109, 16628.

(3) Lin, J.-H.; Elangovan, A.; Ho, T.-I. J. Org. Chem. 2005, 70, 7397.

(4) (a) Anderson, J. D.; McDonald, E. M.; Lee, P. A.; Anderson, M. L.; Ritchie, E. L.; Hall, H. K.; Hopkins, T.; Padias, A.; Thayumanavan, S.; Barlow, S.; Marder, S. R.; Jabbour, G. E.; Shaheen, S.; Kippelen, B.; Peyghambarian, N.; Wightman, R. M.; Armstrong, N. R.; Mash, E. A.; Wang, J. J. Am. Chem. Soc. 1998, 120, 9646. (b) Armstrong, N. R.; Anderson, J. D.; Lee, P. A.; McDonald, E. M.; Wightman, R. M.; Hall, H. K.; Hopkins, T.; Padias, A.; Thayumanavan, S.; Barlow, S.; Marder, S. R. SPIE 1999, 3476, 178. (c) Armstrong, N. R.; Whightman, R. M.; Gross, E. M. Annu. Rev. Phys. Chem. 2001, 52, 391.

(5) (a) Lai, R. Y.; Kong, X.; Jenekhe, S. A.; Bard, A. J. J. Am. Chem. Soc. 2003, 125, 12631. (b) Kapturkiewicz, A. Chem. Phys. 1992, 166, 259. (c) Kawai, M.; Itaya, K.; Toshima, S. J. Am. Chem. Soc. 1980, 84, 2368. (d) Lai, R. Y.; Fleming, J. J.; Merner, B. L.; Vermeij, R. J.; Bodwell, G. J.; Bard, A. J. J. Phys. Chem. A. 2004, 108, 376. (e) Strau $\beta$, J.; Daub, J. Adv. Mater. 2002, 14, 1652.

(6) (a) Goes, M.; Verhoeven, J. W.; Hofstraat, H.; Brunner, K. ChemPhysChem 2003, 4, 349. (b) Thomas, K. R. J.; Lin, J. T.; Tao, Y.-T.; Chuen, C.-H. Chem. Mater. 2002, 14, 3852. (c) Zhu, W.; Hu, M.; Yao, R.; Tian, H. J. Photochem. Photobiol., A 2003, 154, 169. (d) Thomas, K. R. J.; Lin, J. T.; Velusamy, M.; Tao, Y.-T.; Chuen, C.-H. Adv. Funct. Mater. 2004, 14, 83. (e) Chiang, C.-L.; Wu, M.-F.; Dai, D.-C.; Wen, Y.-S.; Wang, J.-K.; Chen, C.-T. Adv. Funct. Mater. 2005, 15, 231. transient absorption and emission measurements and steadystate measurements, the formation of $\mathrm{D}-\mathrm{A}$ type $\mathbf{R E}$ in the singlet excited state $\left({ }^{1} \mathbf{R} \mathbf{E}^{*}\right)$ and/or singlet excimer $\left({ }^{1} \mathbf{R E}{ }_{2}{ }^{*}\right)$ can be attributed to the charge recombination between $\mathbf{R E}^{\bullet+}$ and $\mathbf{R E}^{\bullet-}$ which are generated initially from the radiolytic reaction in Bz. The emission mechanism of $\mathbf{R E}$ in pulse radiolysis strongly depends on the charges resulted from the presence of electron donating- or withdrawing substituents. ${ }^{7}$ In addition, it is found that the presence or absence of excimer emission depends on the geometries of RE.

In this paper, we report the emission from the charge recombination between $\mathbf{R A}^{\cdot+}$ and $\mathbf{R A}^{--}$of $\pi$-conjugated $\mathrm{D}-\mathrm{A}$ type molecules having 9-cyanoanthracenyl as the electron acceptor moiety (RAs); 9-cyano-10-phenylanthracene (PA), 9-cyano-10-phenylethynylanthracene (PEA), and various 9-cyano-10-(donor-substituted phenylethynyl)anthracenes with methoxy- (ie., anisole, OEA), $p$ - $N, N$-(dimethylamino)- (NEA), and $N, N$-di-p-anisylamino- (DEA) groups as donor moieties, because $\mathbf{R A}$ are known as the ECL-active molecules in $\mathrm{CH}_{2} \mathrm{Cl}_{2} \cdot{ }^{3}$ Using pulse radiolysis technique, both $\mathbf{R A}^{\bullet+}$ and $\mathbf{R A}^{\bullet-}$ can be generated at the same time in Bz. Therefore, it is expected that ${ }^{1} \mathbf{R} \mathbf{A}^{*},{ }^{3} \mathbf{R} \mathbf{A}^{*}$, and/or ${ }^{1}(\mathbf{R A})_{2}{ }^{*}$ can be generated from the charge recombination between $\mathbf{R} \mathbf{A}^{\bullet+}$ and $\mathbf{R A}^{\bullet-}$ during the pulse radiolysis of $\mathbf{R A}$ in $\mathrm{Bz}$. The emission from PA with no ethynyl linkage is also examined in order to compare with that from other RA with an ethynyl linkage. The time-resolved transient absorption and emission measurements during the pulse radiolysis of the ECL-active Ms in solution were useful to gain a better understanding of their emission mechanism, providing valuable information for the molecular design with an efficient luminescence character.

\section{Results and Discussion}

Scheme 1 depicts the chemical structures of $\pi$-conjugated D-A type molecules having a 9-cyanoanthracenyl moiety (PA, PEA, OEA, NEA, and DEA). For 9-cyano-10-(donor-substituted phenylethynyl)anthracenes (OEA, NEA, and DEA), their electron-donating character of the substituents (methoxy-, $p-N, N$ (dimethylamino)-, and $N, N$-di- $p$-anisylamino-groups, respectively) increases in this order. ${ }^{3}$

(7) (a) Samori, S.; Hara, M.; Tojo, S.; Fujitsuka, M.; Yang, S.-W.; Elangovan, A.; Ho, T.-I.; Majima, T. J. Phys. Chem. B. 2005, 109, 11735. (b) Samori, S.; Tojo, S.; Fujitsuka, M.; Yang, S.-W.; Elangovan, A.; Ho, T.-I.; Majima, T. J. Org. Chem. 2005, 70, 6661. (c) Samori, S.; Tojo, S.; Fujitsuka, M.; Yang, S.-W.; Ho, T.-I.; Yang, J.-S.; Majima, T. J. Phys. Chem. B 2006, 110, 13296-13303. 
TABLE 1. Photophysical Properties of $\mathrm{RA}$ in $\mathrm{CH}_{2} \mathrm{Cl}_{2}$, Hexane, and $\mathrm{Bz}^{a}$

\begin{tabular}{|c|c|c|c|c|c|c|c|c|c|c|}
\hline \multirow[b]{2}{*}{ RA } & \multicolumn{3}{|c|}{ in $\mathrm{CH}_{2} \mathrm{Cl}_{2}$} & \multicolumn{3}{|c|}{ in hexane } & \multicolumn{4}{|c|}{ in $\mathrm{Bz}$} \\
\hline & $\lambda \max (\mathrm{Abs}), \mathrm{nm}$ & $\lambda \max (\mathrm{Fl}), \mathrm{nm}$ & $\phi_{\mathrm{fl}}$ & $\lambda \max (\mathrm{Abs}), \mathrm{nm}$ & $\lambda \max (\mathrm{Fl}), \mathrm{nm}$ & $\phi_{\mathrm{fl}}$ & $\lambda \max (\mathrm{Abs}), \mathrm{nm}$ & $\lambda \max (\mathrm{Fl}), \mathrm{nm}$ & $\phi_{\mathrm{fl}}$ & $\tau_{\mathrm{fl}}^{b}, \mathrm{~ns}$ \\
\hline PA & $391(411)$ & 455 & 0.28 & $385(496,366)$ & $420(442)$ & 0.56 & $389(413,368)$ & 454 (432) & 0.37 & 6.0 \\
\hline PEA & $421(446)$ & 488 & 0.48 & $415(440)$ & 446 (473) & 0.54 & $422(446)$ & 461 (487) & 0.45 & 5.1 \\
\hline OEA & $431(453)$ & 485 & 0.41 & 455 (484) & 455 (484) & 0.52 & $430(453)$ & $473(500)$ & 0.43 & 4.1 \\
\hline NEA & 482 & 584 & 0.06 & $493(518)$ & 493 (518) & 0.32 & 479 & 557 & 0.19 & 4.0 \\
\hline DEA & 491 & 557 & 0 & 517 & 517 & 0.25 & 490 & 589 & 0.11 & 4.8 \\
\hline
\end{tabular}

${ }^{a}$ Although $\phi_{\mathrm{fl}}$ values for RA have been determined in ref 3, they were found to be incorrect because of a mistake. Therefore, they were carefully redetermined in the present study. The values in parentheses are the maxima of vibrational peaks. ${ }^{b}$ The fluorescence lifetime.

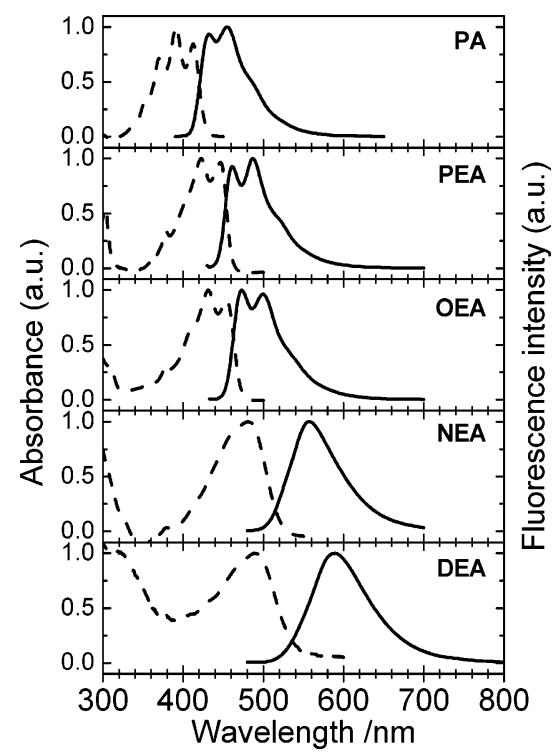

FIGURE 1. Absorption (broken line) and fluorescence (solid line) spectra obtained by the steady-state absorption and fluorescence measurement of $\mathbf{R A}$ in Ar-saturated $\mathrm{Bz}\left(10^{-5} \mathrm{M}\right)$.

Photophysical and Electrochemical Properties. The photophysical properties of $\mathbf{R A s}$ in $\mathrm{CH}_{2} \mathrm{Cl}_{2}(\epsilon=9.08)$, hexane $(\epsilon=1.89)$, and $\mathrm{Bz}(\epsilon=2.28)$ are listed in Table 1. The groundstate absorption spectra of PA, PEA, and OEA in these solvents showed the structured $\pi-\pi^{*}$ transition region around $400-420$ $\mathrm{nm}$ which can be attributed to the local excitation of the 9-cyanoanthracenyl chromophore. On the other hand, those of NEA and DEA clearly showed the structureless band around $480 \mathrm{~nm}$ which can be attributed to the charge-transfer transition. The absorption peaks of RAs in these solvents showed little dependence on the solvent polarity. However, the fluorescence peaks of $\mathbf{R A}$ in $\mathrm{CH}_{2} \mathrm{Cl}_{2}$ or $\mathrm{Bz}$ were observed at longer wavelengths than those in hexane due to the solvent polarity (Table 1 and Figure 1). OEA has been found to have a weak intramolecular charge transfer (ICT) character in the excited state because a red shift was observed in several other solvents such as $\mathrm{Bz}$, THF, $\mathrm{CH}_{2} \mathrm{Cl}_{2}$, acetone, $\mathrm{EtOH}, \mathrm{MeOH}, \mathrm{CH}_{3} \mathrm{CN}$, and DMSO. $^{3}$ On the other hand, the strong ICT is reasonably assumed for NEA and DEA. In Bz, D-A type RA (OEA, NEA, and DEA) also showed a red shift of the emission maxima. Therefore, the ICT is also assumed for D-A type RA in Bz, but not in hexane.

The electrochemical properties of $\mathbf{R A}$ in $\mathrm{CH}_{2} \mathrm{Cl}_{2}$ are listed in Table 2. Using a setup consisting of fluorescence spectrophotometer and voltammograph with a PC interface, ECL emissions of RA were observed with peaks at $456-588 \mathrm{~nm}$ in the presence of $0.05 \mathrm{M}$ tetrabutylammonium perchlorate (TBAP) as supporting electrolyte. ${ }^{3}$ The ECL emission maxima for PA
TABLE 2. Electrochemical Properties of $\mathrm{RA}$ in $\mathrm{CH}_{2} \mathrm{Cl}_{2}{ }^{a}$

\begin{tabular}{lccccc}
\hline & \multicolumn{3}{c}{${\text { in } \mathrm{CH}_{2} \mathrm{Cl}_{2}}$} \\
\cline { 2 - 6 } RA & & & & \multicolumn{2}{c}{$\lambda \max (\mathrm{EC} \mathrm{L})$} \\
\cline { 4 - 6 } & $E_{\mathrm{ox}}, \mathrm{V}$ & $E_{\text {red }}, \mathrm{V}$ & $-\Delta H^{\circ}, \mathrm{eV}$ & $\mathrm{nm}$ & $\mathrm{eV}$ \\
\hline PA & 1.80 & -1.55 & 3.19 & 456 & 2.72 \\
PEA & 1.14 & -1.24 & 2.22 & 552 & 2.25 \\
OEA & 1.10 & -1.32 & 2.26 & 547 & 2.27 \\
NEA & 0.97 & -1.35 & 2.16 & 588 & 2.11 \\
DEA & 0.90 & -1.29 & 2.03 & 643 & 1.93
\end{tabular}

${ }^{a}$ Reference 3 except for the $E_{\text {ox }}$ value of OEA $(1.10 \mathrm{~V})$ and the singlet excited-state energy calculated from the ECL emission maximum of DEA which were measured in this study.

and NEA in $\mathrm{CH}_{2} \mathrm{Cl}_{2}$ (Table 2) are very close to the fluorescence maxima (Table 1).

To elucidate the ECL emission mechanism, the annihilation enthalpy change $\left(-\Delta H^{\circ}\right)$ for the charge recombination between the radical cation $\left(\mathbf{M}^{\bullet+}\right)$ and radical anion $\left(\mathbf{M}^{\bullet}-\right)$ is calculated by eq 1,8

$$
-\Delta H^{\circ}=\left[\left(E_{\mathrm{ox}}-E_{\mathrm{red}}\right)\right]^{\epsilon_{\mathrm{s}}}-\Delta G_{\mathrm{sol}}^{\epsilon_{\mathrm{s}}}-w_{a, \mu}+T \Delta S^{\circ}
$$

where $E_{\mathrm{ox}}$ and $E_{\text {red }}$ are the oxidation and reduction potentials of the solute molecule $(\mathbf{M})$, respectively. $\epsilon_{\mathrm{s}}, \Delta G_{\mathrm{sol}}$, and $w_{a, \mu}$ represent the static dielectric constant of solvent, the free energy change of solvation, and the work required to bring $\mathbf{M}^{\bullet+}$ and $\mathbf{M}^{\bullet-}$ within a likely separation distance, respectively. In $\mathrm{CH}_{2}-$ $\mathrm{Cl}_{2},-\Delta H^{\circ}$ can be expressed using $E_{\mathrm{ox}}$ and $E_{\mathrm{red}}$ measured in $\mathrm{CH}_{2} \mathrm{Cl}_{2}$ by simplified eq $2,{ }^{3}$

$$
-\Delta H^{\circ}=E_{\text {ox }}-E_{\text {red }}-0.16 \mathrm{eV}
$$

The calculated $-\Delta H^{\circ}$ values for $\mathbf{R A}$ are listed in Table 2 . $-\Delta H^{\circ}$ for PA, NEA, and DEA are sufficiently larger than the singlet excited-state energies calculated from the ECL emission maxima, and hence, ${ }^{1} \mathbf{P A}{ }^{*},{ }^{1} \mathbf{N E A} \mathbf{A}^{*}$, and ${ }^{1} \mathbf{D E A}{ }^{*}$ can be generated from the charge recombination between $\mathbf{R A}^{\bullet+}$ and $\mathbf{R A}^{\bullet-}(\mathrm{S}$ route). On the other hand, $-\Delta H^{\circ}$ for PEA and OEA are not sufficiently larger than the energies of the ECL emission maxima. Therefore, it is suggested that initially ${ }^{3} \mathbf{R} \mathbf{A}^{*}$ is generated to be followed by triplet-triplet annihilation giving ${ }^{1} \mathbf{R A} *$ (T-route).

Emission Spectra Observed during the Pulse Radiolysis of $\mathbf{R A}$ in $\mathbf{B z}$. The time-resolved emission spectra were observed to show a monotonic decay after an electron pulse during the pulse radiolysis of RA ( 5 and $10 \mathrm{mM}$ ) in Ar-saturated Bz. At $10 \mathrm{mM}$, all RA showed the emission maxima at $471-680 \mathrm{~nm}$ during pulse radiolysis (Figure 2). For PEA, OEA, and NEA,

(8) Gross, E. M.; Anderson, J. D.; Slaterbeck, A. F.; Thayumanavan, S.; Barlow, S.; Zhang, Y.; Marder, S. R.; Hall, H. K.; Nabor, M. F.; Wang, J.-F.; Mash, E. A.; Armstrong, N. R.; Wightman, R. M. J. Am. Chem. Soc. 2000, 122, 4972 . 


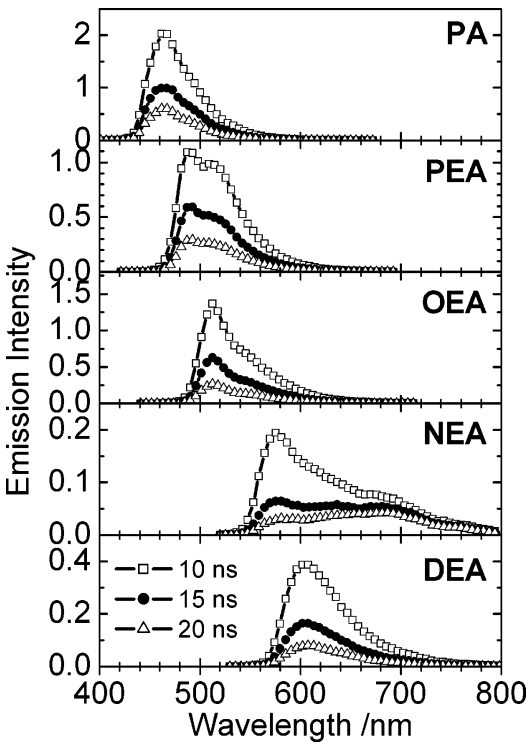

FIGURE 2. Emission spectra recorded at time $t=10$ (open square), 15 (solid circle), and 20 (open triangle) ns after an electron pulse during the pulse radiolysis of $\mathbf{R A}$ in Ar-saturated $\mathrm{Bz}(10 \mathrm{mM})$.

TABLE 3. Emission Maxima $\left(\lambda^{\mathrm{Em}}\right)$ and Relative Emission Intensity during the Pulse Radiolysis of RA in Ar-Saturated Bz (5 and $10 \mathrm{mM}$ )

\begin{tabular}{|c|c|c|c|c|}
\hline \multirow[b]{3}{*}{ RA } & \multicolumn{4}{|c|}{ radiolysis-induced chemiluminescence } \\
\hline & \multicolumn{2}{|r|}{$5 \mathrm{mM}$} & \multicolumn{2}{|r|}{$10 \mathrm{mM}$} \\
\hline & $\lambda^{\mathrm{Em}}, \mathrm{nm}$ & relative intensity $(\%)^{a}$ & $\lambda^{\mathrm{Em}}, \mathrm{nm}$ & relative intensity $(\%)^{a}$ \\
\hline PA & 468 & 100 & 471 & 86.1 \\
\hline PEA & 495 & 49.8 & $491,510^{b}$ & 49.6 \\
\hline OEA & 507 & 38.4 & $513,550^{b}$ & 45.9 \\
\hline NEA & 568 & 15.0 & $576,680^{b}$ & 10.6 \\
\hline DEA & 600 & 12.6 & 608 & 15.8 \\
\hline
\end{tabular}

${ }^{a}$ Relative to the emission intensity for PA in Ar-saturated Bz. Emission intensity was determined from the total amount of emission. ${ }^{b}$ Excimer emission.

another emission maximum was observed at a longer wavelength side during pulse radiolysis and was assigned to the excimer emission, ${ }^{7}$ which was not observed in the steady-state fluorescence measurement even at a high concentration. Because the fluorescence maxima of the D-A type RAs (OEA, NEA, and DEA) were observed at longer wavelength side than that of PEA, an emissive ICT is indicated for the D-A type RAs during pulse radiolysis in Bz. In addition, the shape of the emission spectra of PA, PEA, and OEA were different from those observed in the steady-state measurement and emission maxima observed at the shorter wavelength region disappeared because of the self-absorption as a result of the high concentrations. The radiolysis-induced emission intensities of RA were determined from the total amount of emission and summarized in Table 3. It is assumed that the emissions of RA mainly correspond to 9-cyanoanthracenyl moiety, which is known as an excellent fluorophore. ${ }^{3}$

All RA showed the fluorescence during pulse radiolysis in Bz. It was found that the emission intensity of PA was highest in Ar-saturated Bz. In addition, it was found that the emission intensity of PA at $10 \mathrm{mM}$ was lower than that at $5 \mathrm{mM}$ owing to the strong self-absorption. The emission intensity of NEA at $10 \mathrm{mM}$ was also lower than that at $5 \mathrm{mM}$ owing to the formation of the excimer. On the other hand, the emission intensities of OEA and DEA at $10 \mathrm{mM}$ were higher than those at $5 \mathrm{mM}$

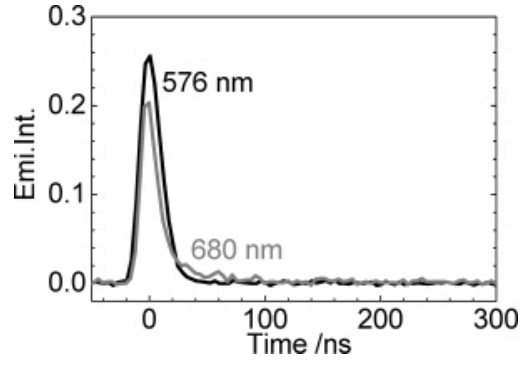

FIGURE 3. Time profiles of the emissions observed during the pulse radiolysis of NEA in Ar-saturated $\mathrm{Bz}(10 \mathrm{mM})$ at 576 and $680 \mathrm{~nm}$.

owing to no intermolecular interaction. For PEA, little change of the emission intensity was observed when the concentration increased. In air-saturated $\mathrm{Bz}$, the emission intensities of all RA decreased.

Figure 3 shows the time profiles of emissions observed at 576 (monomer emission) and $680 \mathrm{~nm}$ (excimer emission) of NEA. It is clearly indicated that the monomer and excimer were formed from the same precursor through the same reaction mechanism. PEA and OEA also showed the same emission behavior.

It was also found that the emission time profiles observed during the pulse radiolysis of $\mathbf{R A}$ in Ar-saturated Bz showed two components: short-lived with high emission intensity and long-lived with very low one. Even at a few microseconds after an electron pulse, the long-lived emission was observed and can be assigned to the delayed fluorescence resulting from triplet-triplet annihilation (T-route)..$^{7 \mathrm{c}}$ The time profiles of emission observed during the pulse radiolysis of RA in airsaturated $\mathrm{Bz}$ (in the presence of dissolved oxygen) showed no long-lived emission component (Figure S1, Supporting Information). It is clearly shown that the long-lived emission component was quenched by oxygen. The quenching was observed for all $\mathbf{R A}$, indicating that the "P-type" delayed fluorescence 9 was confirmed for $\mathbf{R A}$ in $\mathrm{Bz}$ during pulse radiolysis.

Transient Absorption Spectra Observed during the Pulse Radiolysis of RA. Figure 4 shows the transient absorption spectra observed during the pulse radiolysis of RA in $\mathrm{Ar}$ saturated Bz. For D-A type RA (OEA, NEA, and DEA), the absorption bands at around 400-500 nm were not measured due to the strong ground-state absorption. The transient absorption was quenched during the pulse radiolysis of RA in airsaturated Bz. Thus, the transient absorption spectra, observed during the pulse radiolysis of $\mathbf{R A}$ in Ar-saturated $\mathrm{Bz}$, are assigned mainly to ${ }^{3} \mathbf{R A}^{*}$.

From the relationship between $-\Delta H^{\circ}$ and the singlet excitedstate energy calculated from the emission maxima of $\mathbf{R E}$ with the donor-acceptor structure, we have reported that the oneelectron oxidation and reduction of $\mathbf{R E}$ can occur on the donor and acceptor moieties, and that the positive and negative charges of $\mathbf{R E}^{\bullet+}$ and $\mathbf{R E}^{\bullet-}$ are localized on the donor and acceptor moieties, respectively. ${ }^{7}$ Since RA also has the donor-acceptor structure, the same charge localization is expected for $\mathbf{R A}^{\mathbf{}^{+}}$ and $\mathbf{R A}^{\bullet-}$. The absorption spectral shapes of $\mathbf{R A}^{{ }^{+}}$and $\mathbf{R} \mathbf{A}^{\bullet-}$ generated during the pulse radiolysis of RA in Ar-saturated 1,2dichloroethane (DCE) and $N, N$-dimethylformamide (DMF), respectively, clearly indicated such charge localization. It is well

(9) (a) Liu, D. K. K.; Faulkner, L. R J. Am. Chem. Soc. 1977, 99, 4594. (b) Bohne, C.; Abuin, E. B.; Scaiano, J. C. J. Am. Chem. Soc. 1990, 112 4226 . 


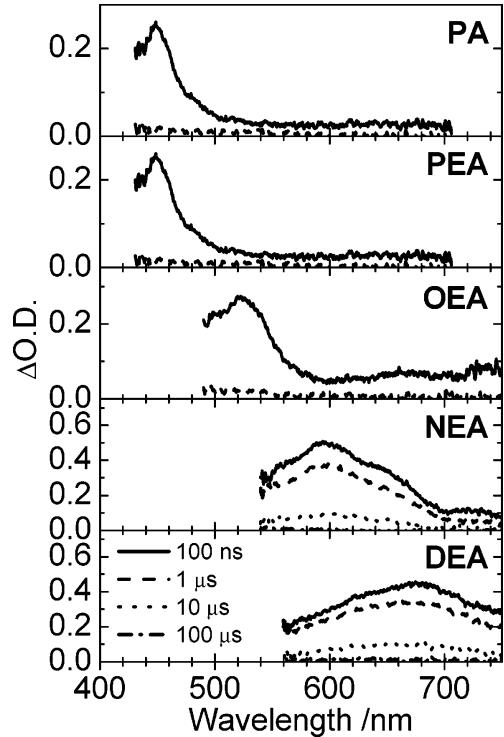

FIGURE 4. Time-resolved transient absorption spectra observed at time $t=100 \mathrm{~ns}, 1,10$, and $100 \mu \mathrm{s}$ after an electron pulse during the pulse radiolysis of $\mathbf{R A}$ in Ar-saturated Bz.

established that $\mathbf{M}^{\bullet+}$ and $\mathbf{M}^{\bullet-}$ are selectively generated during the pulse radiolysis of $\mathbf{M}$ in $\mathrm{DCE}^{10}$ and in $\mathrm{DMF},{ }^{12}$ respectively.

Figure 5 shows the transient absorption spectra observed during the pulse radiolysis of RA in Ar-saturated DCE. The absorption bands observed for all RA with a half-life time on the order of microseconds can be assigned to $\mathbf{R A}^{{ }^{\bullet+}}$. For $\mathbf{P E A} \mathbf{A}^{\bullet+}$, $\mathbf{N E A}^{\bullet+}$, and $\mathbf{D E A}{ }^{\bullet+}$, a new absorption band was observed after the decay of the corresponding radical cation and remained even in the time scale longer than $100 \mu \mathrm{s}$, suggesting that $\mathrm{Cl}^{-}$, generated from the initial radiolytic reactions of DCE, reacts with $\mathbf{R A}^{\bullet+}$ to give the product. ${ }^{7}$ It should be noted that spectral shape of $\mathbf{R A}^{\cdot+}$ depends on molecular structure. Since the absorption spectrum of 9-cyanoanthracene ${ }^{*+}$ with two bands around 400-500 and 700-800 nm (Figure S1(a), Supporting Information) is different from those of $\mathbf{R A}^{\bullet+}$, the electronic property of the donor moiety affects the transient absorption of $\mathbf{R A}^{\cdot+}$, in which the positive charge is not localized on 9-cyanoanthracenyl moiety. For $\mathbf{P A}^{\cdot+}$ and $\mathbf{P E A}{ }^{\bullet+}$, it seems that the positive charge is delocalized on two moieties. For $\mathbf{O E A}{ }^{\bullet+}$, NEA $^{+}$, and $\mathbf{D E A}{ }^{\cdot+}$, two absorption bands were observed in the region of $600-800 \mathrm{~nm}$. The absorption band around 650 $\mathrm{nm}$ corresponds mainly to phenyacetylene ${ }^{\bullet+}$ with an absorption band at $622 \mathrm{~nm} .{ }^{11}$ The other strong absorption peak at 725, 680, and $760 \mathrm{~nm}$ for $\mathbf{O E A}^{\bullet+}, \mathbf{N E A}^{\bullet+}$, and $\mathbf{D E A}{ }^{\bullet+}$, respectively, corresponds to the radical cation of the donor moieties. It has been reported that radical cations of anisole and $N, N^{\prime}$-dimethyl-

(10) (a) Shida, T.; Hamill, W. H. J. Chem. Phys. 1966, 44, 2369, 2375 3472. (b) Shida, T.; Kato, T. Chem. Phys. Lett. 1979, 68, 106. (c) Grimison, A.; Simpson, G. A. J. Phys. Chem. 1968, 72, 1776. (d) Ishida, A.; Fukui, M.; Ogawa, H.; Tojo, S.; Majima, T.; Takamuku, S. J. Phys. Chem. 1995, 99, 10808. (e) Majima, T.; Tojo, S.; Ishida, A.; Takamuku, S. J. Org. Chem. 1996, 61, 7793. (f) Majima, T.; Tojo, S.; Ishida, A.; Takamuku, S. J. Phys. Chem. 1996, 100, 13615

(11) Shida, T. Electronic Absorption Spectra of Radical Ions; Physical Sciences Data 34; Elsevier Science Publishers: Amsterdam, 1988.

(12) (a) Honda, E.; Tokuda, M.; Yoshida, H.; Ogasawara, M. Bull. Chem. Soc. Jpn. 1987, 60, 851. (b) Huddleston, R. K.; Miller, J. R. J. Phys. Chem. 1982, 86, 2410. (c) Majima, T.; Fukui, M.; Ishida, A.; Takamuku, S. $J$. Phys. Chem. 1996, 100, 8913. (d) Majima T.; Tojo, S.; Takamuku, S. J. Phys. Chem. A 1997, 101, 1048.

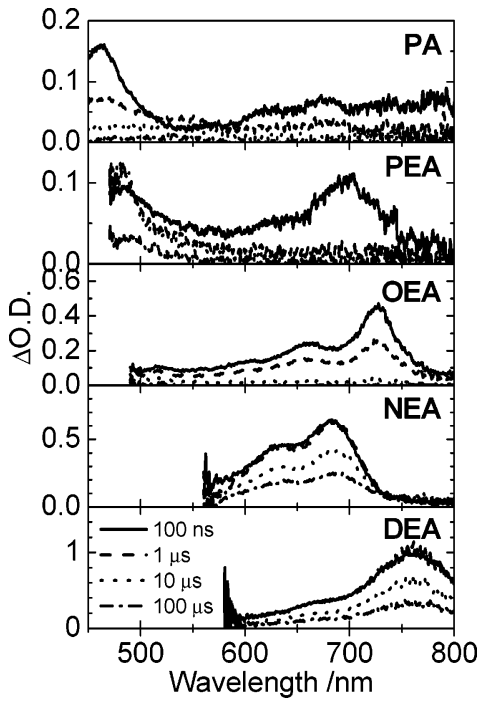

FIGURE 5. Time-resolved transient absorption spectra observed at time $t=100 \mathrm{~ns}, 1,10$, and $100 \mu \mathrm{s}$ after an electron pulse during the pulse radiolysis of RA in Ar-saturated DCE. The sample solutions were prepared in $5 \mathrm{mM}$ concentration.

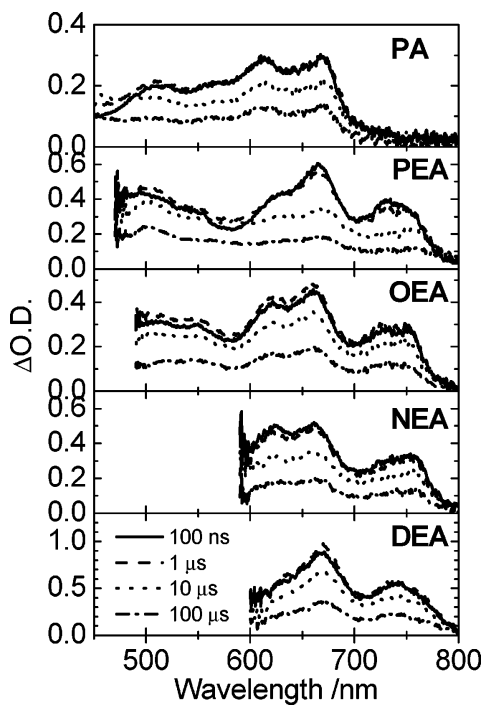

FIGURE 6. Time-resolved transient absorption spectra observed at time $t=100 \mathrm{~ns}, 1,10$, and $100 \mu \mathrm{s}$ after an electron pulse during the pulse radiolysis of RA in Ar-saturated DMF. The sample solutions were prepared in a $5 \mathrm{mM}$ concentration.

aniline have broad and weak absorption bands in the region of 550-1500 and 550-700 nm, respectively. ${ }^{11}$ The lifetimes of PA $^{\cdot+}$ and PEA $^{\cdot+}$ were shorter than one microsecond, while those of $\mathbf{O E A}^{\bullet+}, \mathbf{N E A}^{\bullet+}$, and $\mathbf{D E A}^{\bullet+}$ were in a few microseconds. This suggests that the reactivities of $\mathbf{P A}^{\bullet+}$ and $\mathbf{P E A}{ }^{\bullet+}$ with $\mathrm{Cl}^{-}$are enhanced with increasing cationic character because of the positive charge delocalization including the 9-cyanoanthracenyl moiety in $\mathbf{P A}^{{ }^{+}}$and $\mathbf{P E A}{ }^{\cdot+}$, compared with $\mathbf{O E A}{ }^{\bullet+}$, NEA $^{\bullet+}$, and DEA ${ }^{\bullet+}$.

Figure 6 shows the transient absorption observed during the pulse radiolysis of RA in Ar-saturated DMF. The absorption bands observed for all RA with a half-life time on the order of microseconds can be assigned to $\mathbf{R A}^{\bullet-}$. It should be noted that $\mathbf{P A}^{--}$has the absorption band at $500-700 \mathrm{~nm}$, and other $\mathbf{R A s ^ { - }}$ with the ethynyl linkage have almost the same absorption band at $500-800 \mathrm{~nm}$. It is suggested from the spectral shapes of $\mathbf{R A}^{\mathbf{0}^{-}}$ 
that the negative charges localized mainly on 9-cyanoanthracenyl moiety because 9 -cyanoanthracene ${ }^{--}$has an absorption band at around 500-700 nm (Figure S2(b), Supporting Information). For $\mathbf{R A s}^{{ }^{-}}$- with the ethynyl linkage, the other absorption band appearing at $700-800 \mathrm{~nm}$ corresponds to the ethynyl linkage, because transient absorption spectra of radical anions of $\mathrm{D}-\mathrm{A}$ type 9-cyanoanthracene derivatives without the ethynyl linkage, such as 9-cyano-10-( $p$-anisyl)anthracene, 9-cyano-10- $(p-N, N$ dimethylaminophenyl)anthracene, and 9-cyano-10-( $N, N$-di- $p$ anisylaminophenyl)anthracene, were observed to be similar to that of $\mathbf{P A}^{--}$with no absorption at 700-800 nm during the pulse radiolysis. The lifetimes for all $\mathbf{R A}^{--}$were in the order of several tens of microseconds, suggesting that the negative charges exist on 9-cyanoanthracenyl moiety and the ethynyl linker for $\mathbf{R A}^{\mathbf{-}}$.

No or little emission was observed during the pulse radiolysis of $\mathbf{R A}$ in DCE or DMF, suggesting that $\mathbf{R A}^{\cdot+}$ or $\mathbf{R A}^{\mathbf{}^{-}}$itself does not emit light. In other words, both $\mathbf{R A}^{\bullet+}$ and $\mathbf{R} \mathbf{A}^{\cdot-}$ must be formed at the same time in order to observe the emission. In Bz, however, no transient absorption band of $\mathbf{R A}^{\bullet+}$ and $\mathbf{R A}^{\bullet-}$ was observed immediately after an 8-ns electron pulse. Because $\mathrm{Bz}$ in the singlet excited state $\left({ }^{1} \mathrm{Bz}^{*}\right)$ has a short lifetime (12 $\mathrm{ns})$, the singlet energy transfer to RA is possible only if the energy transfer rate constant is larger than $10^{10} \mathrm{M}^{-1} \mathrm{~s}^{-1}$ even at high RA concentration $(10 \mathrm{mM}) .{ }^{13}$ Since the diffusioncontrolled rate constant in $\mathrm{Bz}$ is known to be $k_{\text {diff }}=1.0 \times 10^{10}$ $\mathrm{M}^{-1} \mathrm{~s}^{-1}$, it is expected that the singlet energy transfer from ${ }^{1} \mathrm{Bz}^{*}$ to $\mathbf{R A}$ is limited only at the $\mathbf{R A}$ concentration larger than $10 \mathrm{mM}$. However, we observed the strong emission within the pulse duration ( $8 \mathrm{~ns}$ ) during the pulse radiolysis of $\mathbf{R A}$ in $\mathrm{Bz}$ at low concentrations of $0.1-5 \mathrm{mM}$. Since the concentrations of generated ${ }^{3} \mathbf{R} \mathbf{A}^{*}$ are estimated to be much lower than $10 \mathrm{mM}$ (approximately $0.01 \mathrm{mM}$ ), emission observed within the 8-ns pulse duration cannot be generated from the triplet-triplet annihilation. Therefore, this emission must correspond to the charge recombination between $\mathbf{R A}^{\bullet+}$ and $\mathbf{R} \mathbf{A}^{\bullet-}$, giving ${ }^{1} \mathbf{R A}^{*}$, ${ }^{3} \mathbf{R A}{ }^{*}$, and ${ }^{1}(\mathbf{R A})_{2}{ }^{*}$ (for PEA, OEA, and NEA) within a pulse duration. ${ }^{7}$ The emission intensity was decreased in the presence of $\mathrm{N}_{2} \mathrm{O}$ and $\mathrm{CH}_{3} \mathrm{OH}$ as the electron and hole scavengers, respectively, indicating that $\mathbf{R A}^{\bullet+}$ and $\mathbf{R A}^{\bullet-}$ are responsible to the emission during the pulse radiolysis of $\mathbf{R A}$ in $\mathrm{Bz}$. It is also notable that ${ }^{1}(\mathbf{R A})_{2}{ }^{*}$ is not generated with the steady-state experiment even at high concentration.

Emission Mechanism. In $\mathrm{Bz},-\Delta H^{\circ}$ can be expressed by simplified eq 3 ,

$$
-\Delta H^{\circ}=E_{\mathrm{ox}}-E_{\mathrm{red}}+0.13 \mathrm{eV}
$$

where $E_{\mathrm{ox}}$ and $E_{\text {red }}$ are the oxidation and reduction potentials of $\mathbf{R A}$ in $\mathrm{CH}_{2} \mathrm{Cl}_{2}$ (Table 2). $-\Delta H^{\circ}$ values for $\mathbf{R A}$ in Bz were shown in Table 4 with the singlet excited-state energy calculated from the emission maxima $\left(E_{\mathrm{S} 1}^{\prime}\right)$ during pulse radiolysis.

Table 4 shows that $-\Delta H^{\circ}$ values for $\mathbf{R A}$ are larger than $E_{\mathrm{S} 1}^{\prime}$, suggesting that the energy available in the charge recombination is sufficient to populate the $S_{1}$ state for all RA. The relation between $-\Delta H^{\circ}$ and $E_{\mathrm{S} 1}^{\prime}$ can explain that the short-lived emission component observed for $\mathbf{R A}$ is resulted from ${ }^{1} \mathbf{R} \mathbf{A}^{*}$ which was formed through the direct charge recombination between $\mathbf{R A}^{\mathbf{}^{+}}$and $\mathbf{R A}^{--}$(S-route), and that the long-lived emission component is from ${ }^{1} \mathbf{R} \mathbf{A}^{*}$ formed via T-route. The relation of $-\Delta H^{\circ}, E_{\mathrm{S} 1}^{\prime}$, and $E_{\mathrm{S} 1}$ (determined at the concentration

(13) Mohan, H.; Brede, O.; Mittal, J. P. J. Photochem. Photobiol., A 2001, 140, 191.
TABLE 4. Annihilation Enthalpy Changes $\left(-\Delta H^{\circ}\right)$ and the Singlet Excited-State Energy Calculated from the Emission Maxima $\left(E_{\text {S1 }}^{\prime}\right)$ during the Pulse Radiolysis of $\mathrm{RA}$ in $\mathrm{Bz}$

\begin{tabular}{lcc}
\hline RA & $-\Delta H^{\circ}, \mathrm{eV}$ & $E_{\mathrm{S} 1}^{\prime}, \mathrm{eV}^{a}$ \\
\hline PA & 3.48 & 2.65 \\
PEA & 2.51 & 2.51 \\
OEA & 2.55 & 2.45 \\
NEA & 2.45 & 2.18 \\
DEA & 2.32 & 2.07
\end{tabular}

${ }^{a}$ Estimated from the peak wavelength of the emission spectra observed during pulse radiolysis $(5 \mathrm{mM})$.

of $10^{-5} \mathrm{M}$ with the steady-state measurements) was discussed in the Supporting Information.

The transient absorption spectral shapes of $\mathbf{P A}^{\bullet+}$ and $\mathbf{P E A} \mathbf{A}^{\bullet+}$ showed that the positive charge is delocalized on two moieties of $\mathbf{R A}^{\bullet+}$, while those of $\mathbf{P A}^{--}$and $\mathbf{P E A}^{--}$showed that the negative charge is localized on 9-cyanoanthracenyl moiety of $\mathbf{R A}^{\bullet-}$ (Figures 4 and 5). Therefore, it is suggested that the charge recombination between $\mathbf{P A}^{\bullet+}$ with delocalized charge and $\mathbf{P A}^{\bullet-}$ with localized one on 9-cyanoanthracenyl moiety occurs to give ${ }^{1} \mathbf{P A}^{*}$ during pulse radiolysis in Bz. It has been indicated by the DFT calculation that PEA has planar geometry owing to the presence of the ethynyl linkage although PA has a twisted geometry. ${ }^{3}$ Thus, for PEA, both $\pi$-stacking interaction between two 9-cyanoanthracenyl moieties and between two phenyl moieties are possible in an encounter complex of PEA with the proximally parallel geometry during the charge recombination between $\mathbf{P E A}^{\bullet+}$ and $\mathbf{P E A}^{\bullet-}$ to give the monomer and excimer emissions (Schemes 2 and 4).

For the D-A type RA, transient absorption spectra of $\mathbf{R A}^{\bullet+}$ showed that the positive charge was localized on the donor moieties. On the other hand, the transient absorption spectra of $\mathbf{R A}^{\bullet-}$ showed that the negative charges of all $\mathbf{R A}^{\bullet-}$ were mainly localized on 9-cyanoanthracenyl moieties. Thus, the emissive ICT state $\left({ }^{1}\left(\mathrm{~A}^{\bullet-}-\mathrm{D}^{\bullet+}\right)^{*}\right)$ can be formed by the charge recombination between $\mathbf{R A}^{\bullet+}$ and $\mathbf{R A}^{\cdot-}$ as shown in Schemes 3 and 4. Therefore, it is suggested that the charge recombination between $\mathbf{R A}^{\bullet+}$ and $\mathbf{R A}^{\bullet-}$ with localized charges occurs to give ${ }^{1} \mathbf{R} \mathbf{A}^{*}$ with the ICT character and ${ }^{1}(\mathbf{R A})_{2}{ }^{*}$ (for OEA and NEA) which emit lights during the pulse radiolysis of D-A type RA in Bz. It has been reported that $\mathrm{D}-\mathrm{A}$ type $\mathbf{R A}$ has twisted geometry. In addition, weak ICT was indicated for ${ }^{1} \mathbf{O E A}^{*}$, and strong ICT was for ${ }^{1} \mathbf{N E A}^{*}$ and ${ }^{1} \mathbf{D E A}{ }^{*}{ }^{3}$ Thus, it is suggested that both $\pi$-stacking interactions between two 9-cyanoanthracenyl moieties and between two $p$-anisyl moieties are possible for the encounter complex of OEA with the proximally parallel geometry during the charge recombination between $\mathbf{O E A}^{\bullet+}$ and $\mathbf{O E A}^{\bullet-}$ to give the monomer and excimer emission. For NEA, it is expected that $\mathbf{N E A}^{\bullet+}$ and $\mathbf{N E A}^{\bullet-}$ collide neck-to-neck to generate the excimer in which two 9-cyanoanthracenyl moieties are stacked in the face-to-face configuration with two dimethylaminophenyl moieties projecting perpendicularly away from each other due to the twisted geometry (Scheme 3). For DEA with strong and bulky donor substituent, a considerably twisted structure is assumed to give ${ }^{1} \mathbf{D E A}^{*}$ with strong ICT character but not ${ }^{1}(\text { DEA })_{2}{ }^{*}$ because of the bulky donor substituent.

Thus, it is clarified that the charge recombination between $\mathbf{R A}^{\bullet+}$ and $\mathbf{R A}^{\bullet-}$ occurs to give ${ }^{1} \mathbf{R} \mathbf{A}^{*}$ and ${ }^{1}(\mathbf{R A})_{2}{ }^{*}$ (for PEA, OEA, and NEA) during the pulse radiolysis of RA in Bz. In addition, the delayed fluorescence (long-lived emission component with very low intensity) was also observed in Arsaturated Bz. This delayed fluorescence was quenched by 


\section{SCHEME 2}
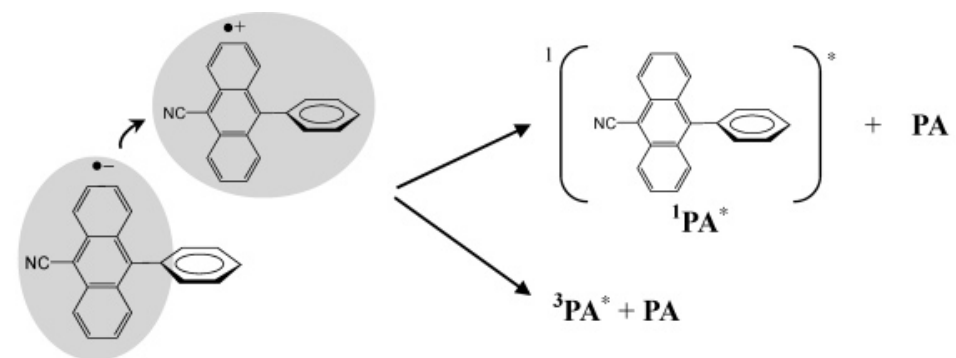

$$
\mathbf{P A}^{\bullet+}+\mathbf{P A}^{\bullet-}
$$
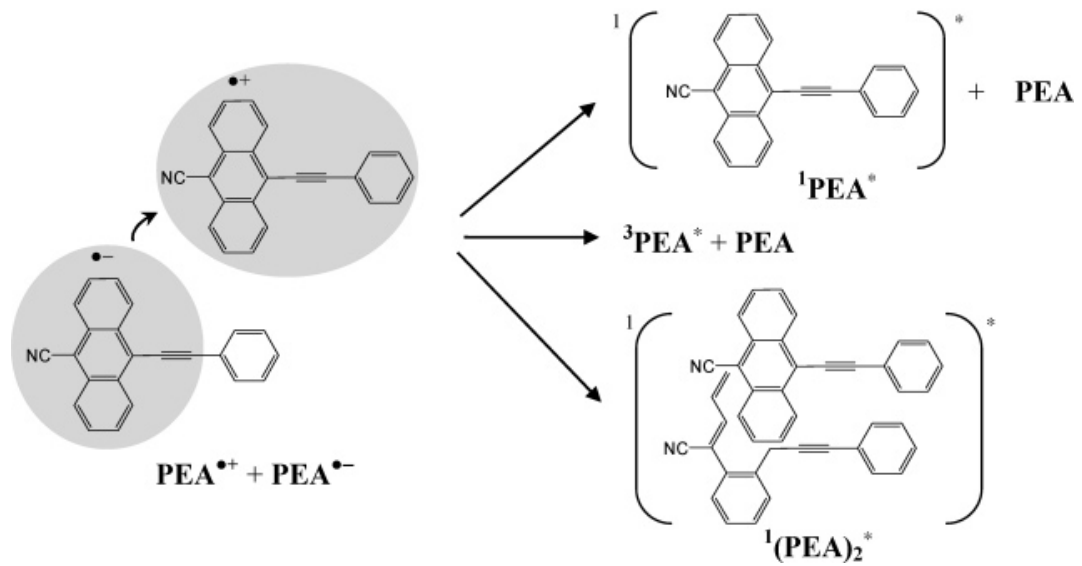

\section{SCHEME 3}
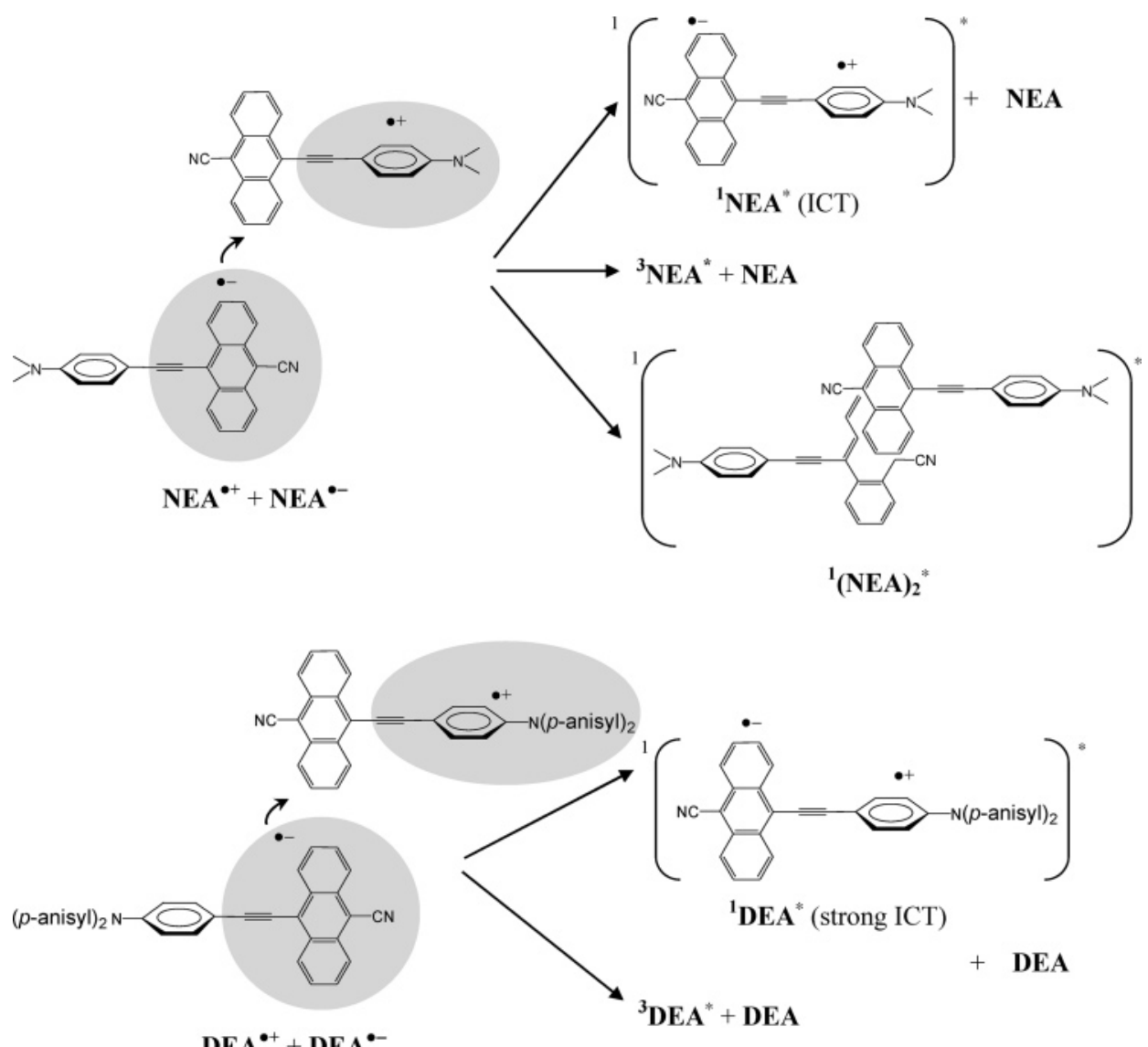

oxygen, indicating that it was derived from the triplet-triplet annihilation (T-route). The mechanism of the delayed fluorescence during the pulse radiolysis of $\mathbf{R A}$ in $\mathrm{Bz}$ is summarized as Scheme 4. The transient absorption spectral shapes of $\mathbf{P A}^{\bullet+}$ and $\mathbf{P E A}{ }^{++}$showed that the positive charge is delocalized on two moieties of $\mathbf{R} \mathbf{A}^{\bullet+}$, while $\mathbf{P A}{ }^{\bullet-}$ and $\mathbf{P E A}{ }^{\bullet-}$ showed that the 
SCHEME 4. Mechanism of the Emission during the Pulse Radiolysis of $\mathbf{D}-\mathbf{A}$ Type $\mathbf{R A}$ in $\mathbf{B z}^{a}$

$$
\begin{aligned}
& \mathrm{e}^{-}+\mathbf{R A} \rightarrow \mathbf{R A}^{\cdot-} \\
& \mathrm{Bz}^{\bullet+}+\mathbf{R A} \rightarrow \mathrm{Bz}+\mathbf{R A}{ }^{\bullet+} \\
& \mathbf{R A}^{\bullet+}+\mathbf{R A}^{\bullet-} \rightarrow{ }^{1} \mathbf{R} \mathbf{A}^{*}+\mathbf{R A} \text { and }{ }^{3} \mathbf{R} \mathbf{A}^{*}+\mathbf{R A} \\
& \mathbf{R A}^{\bullet+}+\mathbf{R A}^{\bullet-} \rightarrow{ }^{1} \mathbf{R A}^{*}+\mathbf{R A},{ }^{3} \mathbf{R A}^{*}+\mathbf{R A}, \text { and }{ }^{1}(\mathbf{R A})_{2}{ }_{2}(\text { for PEA, OEA, and NEA) } \\
& { }^{3} \mathbf{B z}^{*}+\mathbf{R A} \rightarrow \mathbf{B z}^{3}+\mathbf{R A}^{*} \\
& { }^{3} \mathbf{R A}^{*}+{ }^{3} \mathbf{R A}^{*} \rightarrow{ }^{1} \mathbf{R A}^{*}+\mathbf{R A} \\
& { }^{1} \mathbf{R A}^{*} \rightarrow \mathbf{R A}+h v_{\mathrm{f}} \\
& { }^{1} \mathbf{R A}^{*} \rightarrow \mathbf{R A}+h v_{\mathrm{df}} \\
& { }^{1}(\mathbf{R A})_{2}{ }^{*} \rightarrow 2 \mathbf{R A}+h v_{\mathrm{ex}}(\text { for PEA, OEA, and NEA) }
\end{aligned}
$$

\footnotetext{
${ }^{a} h v_{\mathrm{f}}, h v_{\mathrm{ex}}$, and $h v_{\mathrm{df}}$ denote fluorescence from monomer, excimer, and P-type delayed emission derived from triplet-triplet annihilation, respectively. For D-A Type RA, $\mathbf{R A}^{\cdot-}=\mathrm{A}^{\cdot-}-\mathrm{D}, \mathbf{R A}^{\bullet+}=\mathrm{A}-\mathrm{D}^{{ }^{+}},{ }^{1} \mathbf{R} \mathbf{A}^{*}=$ ${ }^{1}\left(\mathrm{~A}^{\cdot-}-\mathrm{D}^{\cdot+}\right)^{*}$
}

negative charge is localized on 9-cyanoanthracenyl moiety of $\mathbf{R A}^{-}$. It is suggested that the positive charge of the D-A type $\mathbf{R A}^{\bullet+}\left(\mathrm{OEA}^{\bullet+}, \mathbf{N E A}^{\bullet+}\right.$, and $\left.\mathbf{D E A}{ }^{\bullet+}\right)$ is localized on donor moieties, and that the negative charge of $\mathbf{R A}^{\cdot-}\left(\mathbf{O E A}^{\bullet-}, \mathbf{N E A}^{--}\right.$, and $\mathbf{D E A}^{--}$) is localized on acceptor moieties. Thus, the charge recombination between $\mathbf{R A}^{{ }^{+}}$and $\mathbf{R A}^{{ }^{-}}$with localized charges occurs to give ${ }^{1} \mathbf{R A}{ }^{*}$ with ICT character and/or ${ }^{1}(\mathbf{R A})_{2}{ }^{*}$ which emit lights during the pulse radiolysis of $\mathrm{D}-\mathrm{A}$ type $\mathbf{R A}$ in $\mathrm{Bz}$.

\section{Conclusions}

Several substituted RA with or without donor-acceptor character showed monomer and excimer emissions during pulse radiolysis in $\mathrm{Bz}$. The emission is suggested to be originated from ${ }^{1} \mathbf{R} \mathbf{A}^{*}$ generated by the charge recombination between $\mathbf{R A}^{\bullet+}$ and $\mathbf{R A}^{\bullet-}$ which are yielded from the initial radiolytic reaction in Bz. This mechanism is reasonably explained by the fact that $-\Delta H^{\circ}$ values $(2.32-3.48 \mathrm{eV})$ estimated for the charge recombination between $\mathbf{R A}^{\bullet+}$ and $\mathbf{R A}^{\bullet-}$ are sufficiently larger than the singlet excited-state energy calculated from the emission maxima ( $\left.E_{\mathrm{S} 1}^{\prime}\right)$ of RA $(2.07-2.65 \mathrm{eV})$. PA and DEA showed only monomer emission during pulse radiolysis in Bz. On the other hand, PEA and NEA showed both monomer and excimer emissions, leading to a reduction in the emission efficiency.

Although the introduction of the ethynyl linkage for RA provides the broad emission which covers the visible region, that also causes aggregation to form excimer, leading to a reduction in the emission efficiency. However, RA with a strong electron donor substituent (DEA) exhibits strong ICT $\left({ }^{1}\left(\mathrm{~A}^{\bullet--}\right.\right.$ $\left.\left.\mathrm{D}^{\bullet^{+}}\right)^{*}\right)$ and the excimer structure is not formed due to the twisted geometry, precluding reduction in the emission efficiency even at high concentration. The emission property of this type of $\pi$-conjugated D-A type molecule can be useful information for developing electroluminescent materials for OLEDs.

\section{Experimental Section}

Measurements of Photophysical and Electrochemical Properties. UV spectra were recorded in Bz with a UV/visible spectrometer using a transparent rectangular cell made from quartz $(1.0 \times 1.0$ $\times 4.0 \mathrm{~cm}$, path length of $1.0 \mathrm{~cm}$ ). Fluorescence spectra were measured with a spectrofluorometer. The oxidation and reduction potential values were measured with cyclic voltammograms $(\mathrm{CV})$. Using a setup consisting of a spectrofluorometer and voltammograph with a PC interface, ECL emissions were measured. ${ }^{2,3}$ The fluorescence quantum yields $\left(\phi_{\mathrm{fl}}\right)$ were determined by using coumarin 106 standard $\left(\phi_{\mathrm{fl}}=0.57\right.$ in $\left.\mathrm{MeOH}, \lambda_{\mathrm{ex}}=390 \mathrm{~nm}\right)$. The fluorescence lifetime $\left(\tau_{\text {fl }}\right)$ measurement was carried out using a single photon counter.

Pulse Radiolysis. Pulse radiolysis experiments were performed using an electron pulse ( $28 \mathrm{MeV}, 8 \mathrm{~ns}, 0.87 \mathrm{kGy}$ per pulse) from a linear accelerator at Osaka University. All the sample solutions were prepared in a 5 and $10 \mathrm{mM}$ concentration in $\mathrm{Bz}$ in a rectangular quartz cell $(0.5 \times 1.0 \times 4.0 \mathrm{~cm}$, path length of 1.0 $\mathrm{cm})$. These solutions were saturated with Ar gas by bubbling with it for $10 \mathrm{~min}$ at room temperature before irradiation. The kinetic measurements were performed using a nanosecond photoreaction analyzer system. The monitor light was obtained from a pulsed 450-W Xe arc lamp, which was operated by a large current pulsedpower supply that was synchronized with the electron pulse. The monitor light was passed through an iris with a diameter of $0.2 \mathrm{~cm}$ and sent into the sample solution at a perpendicular intersection to the electron pulse. The monitor light passing through the sample was focused on the entrance slit of a monochromator and detected with a photomultiplier tube. The transient absorption and emission spectra were measured using a photodiode array with a gated image intensifier as a detector. To avoid pyrolysis of the sample solution by monitor light, a suitable cutoff filter was used.

Acknowledgment. We thank the members of the Radiation Laboratory of SANKEN, Osaka University, for running the linear accelerator. This work has been partly supported by a Grant-in-Aid for Scientific Research (Project 17105005, Priority Area (417), 21st Century COE Research, and others) from the Ministry of Education, Culture, Sports, Science and Technology (MEXT) of the Japanese Government.

Supporting Information Available: Time profiles of the emission intensities at $576 \mathrm{~nm}$ observed during the pulse radiolysis of NEA in Ar- and air-saturated Bz $(5 \mathrm{mM})$. Transient absorption spectra observed during the pulse radiolysis of 9-cyanoanthracene $(5 \mathrm{mM})$ in Ar-saturated DCE, and DMF. The relation of $-\Delta H^{\circ}$, $E_{\mathrm{S} 1}$, and $E_{\mathrm{S} 1}^{\prime}$ values. This material is available free of charge via Internet at http://pubs.acs.org.

JO060929N 